\section{Laparoscopic staging of borderline ovarian tumours - Technique and results}

\author{
Klimatsouda M, Donoudis C, Kontopoulos G and Daponte A* \\ Department of Obstetrics and Gynecology, Faculty of Medicine, School of Health Sciences \\ University of Thessaly, University Hospital of Larisa, Greece
}

\section{Abstract}

Borderline Ovarian Tumors (BOT) tend to present more frequently nowadays, especially in younger women. Furthermore fertility preservation and laparoscopic management is often desired and therefore appropriate counselling is challenging and the treatment selection must be made on evidence based medicine.

Adnexal mass could be a random finding when a typical gynecologic examination is performed. The diagnostic algorithm for possible BOT is the same as for any ovarian tumor, but the treatment options and techniques may vary based on patient's willing to preserve her fertility or not. Laparoscopic or laparotomy approach has similar results although intraoperative findings and frozen section may redirect the primary treatment planning.

When an initial conservative approach is chosen, a secondary approach including total hysterectomy and bilateral salpingo-oophorectomy with staging should be considered. Hence a full counselling is recommended before any primary approach.

\section{General aspects}

Borderline ovarian tumors were first described by Taylor in 1929. The average age of women with Borderline ovarian tumors is 40 years. The percentage of ovarian tumors which are classified histologically as borderline is between 10 to $20 \%$. They are encountered usually in one ovary (unilateral). When found bilateral they are in $30 \%$ serous and in $8 \%$ mucinous.

The symptoms of BOT are the same as for any adnexal mass and include irregular menses, pain or pressure in the lower abdomen, urinary frequency or constipation and sometimes dyspareunia. Many times, the mass is found incidentally during the routine gynecologic examination.

BOT are usually diagnosed in an early stage and they have a good prognosis -except from the ones with micropapillary or clear cell histology, but they are often misdiagnosed and suboptimal operated, whereas surgical restaging is always required. BOT are associated with age, nulliparity and probably with use of ovarian stimulating drugs [1,2].

In BOT, pathological classification is important, especially for high-risk subtypes. borderline tumours. to be clarified.
More Information

*Address for Correspondence: Daponte A, Department of Obstetrics and Gynecology, Faculty of Medicine, School of Health Sciences University of Thessaly, University Hospital of Larisa, Greece, Tel: +302413502795; +302413502796 Email: daponte@med.uth.gr; dapontealexandros@gmail.com; maryklim79@gmail.com

\section{Submitted: February 24, 2021}

Approved: March 16, 2021

Published: March 17, 2021

How to cite this article: Klimatsouda M Donoudis C, Kontopoulos G and Daponte A Laparoscopic staging of borderline ovarian tumours - Technique and results. Clin J Obstet Gynecol. 2021; 4: 020-024.

\section{DOI: 10.29328/journal.cjog.1001081}

Copyright: @ 2021 Klimatsouda M, et al. This is an open access article distributed under the Creative Commons Attribution License, which permits unrestricted use, distribution, and reproduction in any medium, provided the original work is properly cited.

Keywords: Borderline; Ovarian tumor; Laparoscopy; Staging; Fertility preservation

Abbreviations: BOT: Borderline Ovarian Tumor; Ca 125: Cancer antigen 125; IOTA LR2: International Ovarian; Tumor Analysis LR2 risk model; MRI: Magnetic Resonance Imaging; TAH: Total Abdominal Hysterectomy; BSO: Bilateral Salpingo-oophorectomy; TVS: Transvaginal Sonography

(2) Check for updates

(6) OPEN ACCESS

In order to clarify this possible association ovarian cancer of different histological types and borderline it is recommended that ovarian tumors should be analysed separately to obtain a more reliable difference in incidence between cancer and

Currently, no 'safe' limits on dose or duration of any of the other drugs used in ovarian stimulation are recommended. One important question for women and practitioners to be determined is whether clomiphene citrate alone is less likely to cause cancer compared with multi-therapy; also, risks associated with the number of IVF stimulation cycles remain

The diagnosis of BOT requires a full medical history, physical examination, pelvic and abdominal imaging and surgical evaluation. The final diagnosis necessitates a histological confirmation. 
The diagnosis requires transvaginal ultrasound ideally by an experienced specialist and biomarker Ca 125 [3]. Often the ultrasound's images are similar with benign or invasive tumours. BOT histology is usually associated within a mass with both solid and fluid components, unilocular cyst or with papilla, which could be seen with an invasive tumor. As recently reported, the IOTA LR2 model for the ultrasound has a similar sensitivity to MRI for discriminating between benign and malignant tumors and a higher specificity compared with MRI. Either alone or in conjunction with MRI, the IOTA LR2 model should be included in preoperative evaluation of adnexal masses [4].

Nevertheless, only tumor size and high levels of Ca 125 could set the suspicion of ovarian malignancy. Tumor size $>8 \mathrm{~cm}$ is associated with $22,4 \%$ invasive cancer on final pathology vs. 3,2\% in tumors $<8 \mathrm{~cm}$ [5].

\section{Surgical evaluation - Treatment}

The intraabdominal surgical procedure should be the same either with open or laparoscopic surgery and this must be preoperatively explained to the patient.

The surgical evaluation and treatment are based upon age and desire of fertility preservation. In cases where either fertility preservation is not desired or family is completed the surgical approach is staging laparotomy or laparoscopy including total hysterectomy with bilateral salpingooophorectomy, omentectomy, peritoneal washing and multiple peritoneal biopsies. Only suspicious lymph nodes should be sampled. Appendectomy may be considered if the histological type reveals mucinous type. This is an additional reason why intraoperative frozen section and histologic examination is always required.

If fertility preservation or endocrine function preservation is required (stage I) cystectomy or unilateral salpingooophorectomy could be the surgical option. Still in those cases full staging should be performed; pelvic washings should be sampled with omental biopsy and biopsies of any peritoneal lesions. Appendectomy should be again considered in cases of mucinous type BOT. Furthermore, clear cell should be excluded from conservative management. Intraoperative Frozen section and histologic examination is required.

The dilemma that arises is if simple cystectomy or oophorectomy or unilateral salpingo-oopherectomy (USO), is an appropriate treatment for women with BOTs who wish to preserve fertility. Even a cystectomy could be an acceptable fertility-sparing therapy in certain patients, when a cystectomy is the only surgical option after appropriate extensive counselling. Even in advanced disease with one ovary unaffected, fertility may be retained provided the patient gets full counselling.

In each patient, regardless if fertility preservation is intended or not, thorough abdominal inspection for macroscopic findings should be performed. In particular, the surgeon overviews thoroughly for presence of extraovarian implants, exophytic growth, anarchic vascularization on the surface of the ovary or ascites.

There is an ongoing debate, for more than ten years, whether laparoscopic or open surgery approach is preferable for BOTs treatment.

Criteria for referral to gynaecological oncologist are subcategorized into two groups, premenopausal $(<50 \mathrm{yrs})$ and postmenopausal ( $>50 \mathrm{yrs}$ ) as below [6].

\begin{tabular}{|c|c|}
\hline Premenopausal & Postmenopausal \\
\hline CA 125 levels (> $200 \mathrm{iu} / \mathrm{ml})$ & Elevated CA 125 levels \\
\hline Ascites & Ascites \\
\hline $\begin{array}{l}\text { Evidence of abdominal or distant } \\
\text { metastasis }\end{array}$ & Nodular or fixed pelvic mass \\
\hline \multirow[t]{2}{*}{$\begin{array}{l}\text { Family history of breast or ovarian } \\
\text { Cancer }\end{array}$} & $\begin{array}{l}\text { Evidence of abdominal or distant } \\
\text { metastasis }\end{array}$ \\
\hline & Family history of breast or ovarian cancer \\
\hline
\end{tabular}

As far as intraoperative frozen section is concerned sensitivity varies from $71,1 \%$ to $75 \%$, positive predictive value ranges from $84,3 \%$ to $94,5 \%$. It must be mentioned that BOTs are not only often under diagnosed in $6,6 \%$ to $28 \%$, but also over diagnosed in up to $30,6 \%$ of BOT patients [5].

When intraoperative frozen section reveals endometrioid or clear cell tumor's histologic subtype reclassification in final histology is obligatory [5].

Tumor size can be associated with the likelihood of invasive cancer on final histopathological examination of the specimen. BOTs over $8 \mathrm{~cm}$ are related to invasiveness up to $22,4 \%$. On the other hand, size $<8 \mathrm{~cm}$, are less likely to reveal invasive cancer with a reported incidence of $3,2 \%$. Hence, comprehensive surgical staging in BOT $>8 \mathrm{~cm}$ in diameter, as well as those with micropapillary serous, endometrioid, and clear cell histology diagnosed at the time of frozen section analysis is essential $[5,7]$.

Intraoperative frozen section is also useful to differentiate BOTs and benign tumors but concerning differential diagnosis between BOTs and epithelial ovarian cancer it must be remembered that it is not always feasible with frozen section!

\section{What about lymph nodes?}

Lymph node sampling has low prognostic utility. The overall survival in BOT patients is $98 \%$ for 6,5 years [22]. In BOT the involvement of lymph nodes is $17-21 \%$, whereas malignant transformation is about $2 \%$. For advanced stage BOT tumors lymph node involvement can't be used as a prognostic factor [9].

Several studies have shown that we can omit lymphadenectomy, even for the advanced disease because there is no difference in recurrence or survival rate $[8,10-12]$. 


\section{Technique}

Omentectomy is a standard procedure for the surgical management of BOTs. The performance of a laparoscopic omentectomy includes in laparoscopic staging the set of 3 ports. The usual position of these ports are a $5 \mathrm{~mm}$ port in the right side of the abdomen, a $10 \mathrm{~mm}$ camera port usually in the umbilicus and a $10 \mathrm{~mm}$ port in the left side of the abdomen. When we perform a partial omentectomy, we remove only certain part of the omentum. More specifically for laparoscopic staging in BOT cases, we prefer infracolic omentectomy; arcades of the gastroepiploic vessels are preserved and the range of resection is bellow gastroepiploic arcades and the rest of the gastrocolic ligament. We make a dissection line 4-5 cm apart from the greater curvature of the stomach. The omentum is divided $2-3 \mathrm{~cm}$ distance from the gastroepiploic vessels. We make a window at $2-3 \mathrm{~cm}$ apart from the arc of the gastroepiploic vessels near lower to mid body of the stomach. Operator's left hand grasps the greater curvature of the stomach and lifts upward direction, while operator's right hand divides omentum following the gastroepiploic vessels towards the left side to the lower pole of the spleen. It is important to identify the colon to prevent its injury and make sure that the colon wall is not in the way of the dissecting plane. The left gastroepiploic vessels are ligated as the same method as used for total omentectomy [13].

The requirements for a successful laparoscopic surgery are a skilled surgeon, mass $<5 \mathrm{~cm}$, removal of the affected ovary (if conservative therapy desired), cystectomy if fertility is desired in cases of bilateral neoplasia. It must be remembered that cystectomy is reported with a higher risk of relapse [14].

The advantages of laparoscopic techniques include; the use of magnification enables a thorough complete examination of the abdominal cavity to confirm diagnosis and facilitate appropriate staging, facilitation of aspiration and washing of the peritoneum, minimization of surgical invasiveness, postoperative pain and wound - related complications $[15,16]$.

On the other hand, complications of laparoscopic techniques are; possibility of undertreatment (reported associations with higher risk of recurrence and lower survival rates), port site metastasis and most important rupture of the cyst.

\section{Concerns}

Fertility sparing surgery should be recommended with caution.

Advanced stage ( $\geq$ stage II), a serous type, and the presence of bilateral and micropapillary lesions were associated with a higher recurrence rate and a shorter recurrence interval after conservative surgery.

Treating physicians must be careful in choosing fertility sparing surgery patients and pregnancy should be advised as soon as possible after the surgery. Patients that are not yet ready to conceive can be referred to reproductive endocrinology and infertility clinics for other fertility options, including the freezing of embryos and oocytes.

Adnexectomy (unilateral salpingo-oophorectomy) is preferable for patients with unilateral borderline ovarian tumors to achieve ideal oncological outcomes and a satisfactory pregnancy rate.

Bilateral cystectomy is preferably chosen for patients with bilateral borderline ovarian tumors, since no statistically significant difference in terms of recurrence rate, DFS or pregnancy outcome was observed between bilateral cystectomy and unilateral salpingo-oophorectomy + contra lateral cystectomy groups [14].

Short follow-up periods should be advised, in order to investigate the outcomes of borderline ovarian tumor patients undergoing fertility sparing surgery [14].

\section{Surgical restaging after final histology - exceptions}

Surgical restaging is required when initial staging is missing. Laparoscopy in expert hands could still be a valuable surgical option. A selected group of patients could be exempted from restaging; age $>30 \mathrm{yrs}$, radical treatment, stage IA, negative peritoneal cytology, mucinous- diploid- non micropapillary BOT [17].

In conclusion, the management of BOT could be summarized in the following figure 1.

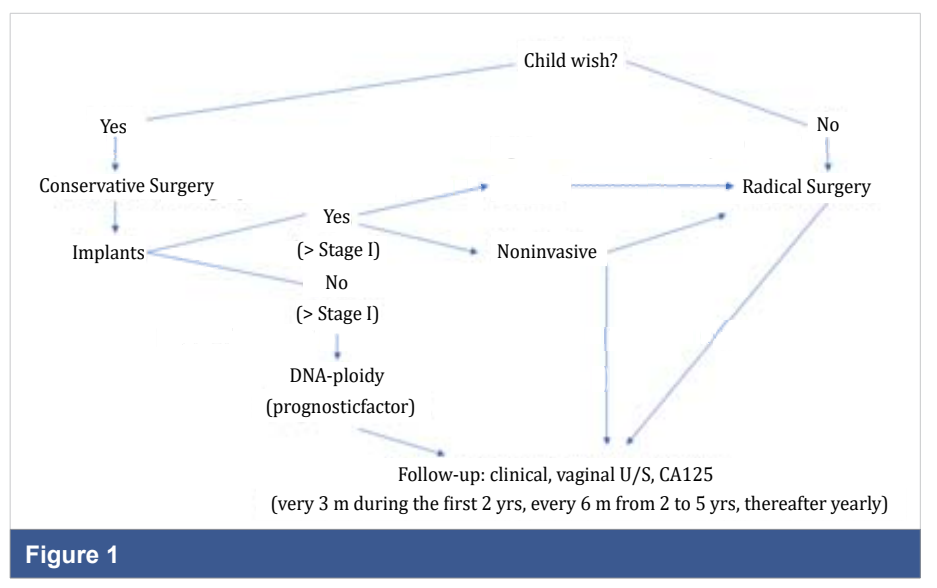

Conservative: Preferable unilateral Salpingo-oophorectomy or cystectomy when Bilateral tumor/Unilateral salpingo oophorectomy in Medical History.

\section{Radical: $T A H+B S O$}

Common Steps: Peritoneal washings, Omentectomy, Multiple peritoneal biopsies and resection of implants. Appendectomy (mucinous).

In summary, we present the following practice points which should be considered by gynecology-oncologists, as far as BOTs are concerned. 
- Borderline ovarian tumours present similarly to other types of adnexal masses.

- Surgery is the primary treatment, and the surgical approach-open versus minimally invasive-should be individualized.

- For women in the reproductive age group, preoperative fertility preservation counselling is recommended.

- Since most borderline tumours are confined to the ovary (ies) and may involve one ovary, fertility-sparing surgery is feasible in a high proportion of women in the reproductive age group.

- Even if bilateral ovarian involvement is noted, fertilitysparing surgery may still be feasible with bilateral ovarian cystectomies or ovarian cystectomy plus unilateral salpingo-oophorectomy.

If borderline ovarian tumor is diagnosed on frozen section examination, surgical staging should include cytologic washings, omentectomy and peritoneal biopsies.

Routine lymphadenectomy is not recommended as part of surgical staging.

If extra-ovarian spread is noted at surgery, maximum cytoreductive surgery with no gross residual should be the objective.

Postoperative treatment is recommended only for those patients with serous borderline tumours and invasive peritoneal implants.

If relapse with borderline tumor occurs, standard treatment consists of surgery alone.

If relapse with invasive carcinoma occurs, treatment may include secondary surgery and chemotherapy [18-25].

\section{Discussion}

BOTs are more likely to present in younger women; therefore, it is important to choose the best treatment whether fertility preservation is desired.

Borderline ovarian epithelial neoplasms are noninvasive neoplasms that occasionally have intraperitoneal spread. The majority of cases are serous or mucinous. If early diagnosed in stage 1 , the five-year survival rate approaches $99 \%$. The clinical presentation of Borderline Ovarian Tumors is the same as it is for other adnexal masses, while occasionally could be a random ultrasound finding in examination per year. The diagnostic approach includes a thorough medical history, oncology family history, infertility treatments, a physical examination, pelvic and abdominal imaging and surgical evaluation. Tumor size and serum CA 125 have a diagnostic value, but are not pathognomonic with low positive predictive value. The complete staging procedure for any suspicious ovarian mass is the same and can be carried out laparoscopically or by laparotomy dependent on the surgeons skill and preference to achieve optimal treatment and staging for his patient [26]. The surgical treatment of BOTs depends on the age of the patient, their reproductive status, the surgical findings (stage, frozen section's results etc.) and fertility preservation. In postmenopausal women and when fertility preservation is not desired a full staging procedure with Total Abdominal Hysterectomy and Bilateral Salpingo- Oophorectomy (TAHBSO), omentectomy and peritoneal washings is performed. In women who wish fertility preservation, a conservative treatment approach can be done when results demonstrate disease stage I and patients are willing to close follow up by the gynecologist. Frozen section must be performed intraoperative and the results are used to determine the surgical approach. Lymphadenectomy has a low prognostic or therapeutic utility in patients with borderline tumors. The overall risk of recurrence after conservative surgery ranges from $7 \%$ to $30 \%$. Even in conservative surgery for fertility preservation, omentectomy retains its significance for prognosis and incomplete surgical staging has been recently confirmed to be an independent negative prognostic factor for disease recurrence [27-29]. The recurrence rate is higher after cystectomy as well as after laparoscopic approach, although there is no difference between laparotomy and laparoscopy in a 20-year follow up. Chemotherapy is rarely indicated for women with borderline ovarian tumors. Follow-up program includes clinical examination, TVS, levels of CA 125, primarily every 3 months during the first 2 years, every 6 months from 2 to 5 years and thereafter yearly [31].

The consultant should counsel the patient about different surgical approaches laparoscopy versus laparotomy, risks, the influence of radicality in surgery and possible recurrence rates, fertility preservation treatment options, additional measures for malignancy should include chemotherapy, follow up schedule and secondary surgical procedures after family planning is completed [30]. The primary treatment planning could be redirected due to surgical findings, frozen section's results etc. That could include surgical restaging and oncological counselling. Therefore, patient's full consent is necessary for treatment planning. A concession form should be signed.

\section{References}

1. Rizzuto I, Behrens RF, Smith LA. Risk of ovarian cancer in women treated with ovarian stimulating drugs for infertility. Cochrane Database Syst Rev. 2013: CD008215.

PubMed: https://pubmed.ncbi.nlm.nih.gov/23943232/

2. Ness RB, Cramer DW, Goodman MT, Kjaer SK, Mallin K, et al. Infertility, fertility drugs, and ovarian cancer: a pooled analysis of casecontrol studies. Am J Epidemiol. 2002; 155: 217-224.

PubMed: https://pubmed.ncbi.nlm.nih.gov/11821246/

3. Kimura E, Murae M, Koga R, Odawara Y, Nakabayashi Y, et al [Clinical significance of new tumor marker CA 125 in gynecological cancer--particularly usefulness in diagnosis of ovarian cancer]. Nihon Sanka Fujinka Gakkai Zasshi. 1984; 36: 2121-2128.

PubMed: https://pubmed.ncbi.nlm.nih.gov/6595320/ 
4. Shimada K, Matsumoto K, Mimura T, Ishikawa T, Munechika J, et al. Ultrasound-based logistic regression model LR2 versus magnetic resonance imaging for discriminating between benign and malignant adnexal masses: a prospective study. Int J Clin Oncol. 2018; 514-521. PubMed: https://pubmed.ncbi.nlm.nih.gov/29236181/

5. Tropé CG, Kaern J, Davidson B. Borderline ovarian tumours. Best Pract Res Clin Obstet Gynaecol. 2012; 26: 325-336. PubMed: https://pubmed.ncbi.nlm.nih.gov/22321906/

6. Im SS, Gordon AN, Buttin BM, Leath CA, Gostout BS, et al. Validation of Referral Guidelines for Women with Pelvic Masses. Obstet Gynecol. 2005; 105: 35-41.

PubMed: https://pubmed.ncbi.nlm.nih.gov/15625139/

7. Park JY, Kim DY, Kim JH, Kim YM, Kim KR, et al. Micropapillary pattern in serous borderline ovarian tumors: does it matter? Gynecol Oncol. 2011; 123: 511-516.

PubMed: https://pubmed.ncbi.nlm.nih.gov/21917305/

8. Seidman JD, Kurman RJ. Ovarian serous borderline tumors: a critica review of the literature with emphasis on prognostic indicators. Human Pathol. 2000; 31: 539-557.

PubMed: https://pubmed.ncbi.nlm.nih.gov/10836293/

9. Lesieur B, Kane A, Duvillard P, Gouy S, Pautier P, et al. Prognostic value of lymph node involvement in ovarian serous borderline tumors. Am J Obstet Gynecol. 2011: 204: e1-7.

PubMed: https://pubmed.ncbi.nlm.nih.gov/21349494/

10. Desfeux P, Camatte S, Chatellier G, Blanc B, Querleu D, et al. Impact of surgical approach on the management of macroscopic early ovarian borderline tumors. Gynecol Oncol. 2005; 98: 390-395.

PubMed: https://pubmed.ncbi.nlm.nih.gov/16043215/

11. Moroney MR, Berning AA, Sheeder J, Corr BR. An Evaluation of Frozen Section and Lymph Node Dissection results for Mucinous Ovarian Tumors. Int J Gynecol Cancer. 2018; 28: 92-98.

PubMed: https://pubmed.ncbi.nlm.nih.gov/29194190/

12. Camatte $S$, Morice $P$, Atallah D, Thoury A, Pautier $P$, et al. Clinica outcome after laparoscopic pure management of borderline ovarian tumors: results of a series of 34 patients. Ann Oncol. 2004; 15: 605-609. PubMed: https://pubmed.ncbi.nlm.nih.gov/15033667/

13. Nezhat FR, Pejovic T, Finger TN, Khalil SS. Role of minimally invasive surgery in ovarian cancer. J Minim Invasive Gynecol. 2013; 20: 754-765. PubMed: https://pubmed.ncbi.nlm.nih.gov/24183269/

14. Fang C, Zhao L, Chen X, Yu A, Xia L, et al. The impact of clinicopathologic and surgical factors on relapse and pregnancy in young patients ( $\leq 40$ years old) with borderline ovarian tumors. BMC Cancer. 2018; 18: 1147.

PubMed: https://pubmed.ncbi.nlm.nih.gov/30463533/

15. Sánchez J, Rosado R, Ramírez D, Medina P, Mezquita S, et al. Torsion of the greater omentum: treatment by laparoscopy. Surg Laparosc Endosc Percutan Tech. 2002; 12: 443-445.

16. Gul YA, Jabbar MF, Moissinac K. Primary torsion of the greater omentum. Acta Chir Belg. 2001; 101: 312-314.

PubMed: https://www.ncbi.nlm.nih.gov/pubmed/11868511

17. Azuar AS, Bourdel N, Ferrarrese G, Dauplat J, Mage G, et al Laparoscopic restaging of borderline ovarian tumours (BLOT): a retrospective study of 142 cases. Eur J Obstetr Gynecol Reprod Biol. 2013; 168: 87-91.

PubMed: https://pubmed.ncbi.nlm.nih.gov/23395007/

18. David M. Gershenson. Management of Borderline Ovarian Tumours Best Pract Res Clin Obstet Gynaecol.

PubMed: https://pubmed.ncbi.nIm.nih.gov/27780698/
19. Huang $\mathrm{Y}$, Zhang W, Wang $\mathrm{Y}$. The feasibility of fertility-sparing surgery in treating advanced-stage borderline ovarian tumors: A meta-analysis, Taiwanese J Obstet Gynecol. 2016; 55: 319-325.

PubMed: https://pubmed.ncbi.nlm.nih.gov/27343308/

20. Jiao X, Hu J, Zhu L. Prognostic Factors for Recurrence After FertilityPreserving Surgery in Patients With Borderline Ovarian Tumors .A Systematic Review and Meta-analysis of Observational Studies. Int J Gynecol Cancer. 2017; 27: 1833-1841.

PubMed: https://pubmed.ncbi.nlm.nih.gov/28816706/

21. Qian X, Hua X, Wu J, Shen Y, Cheng X, et al. Clinical Predictors of Recurrence and Prognostic Value of Lymph Node Involvement in the Serous Borderline Ovarian Tumor. Int J Gynecol Cancer. 2018: 28: 279-284.

PubMed: https://pubmed.ncbi.nlm.nih.gov/29194193/

22. Vasconcelos I, de Sousa Mendes M. Conservative surgery in ovarian borderline tumours: A meta-analysis with emphasis on recurrence risk Eur J Cancer. 2015: 51: 620-631.

PubMed: https://pubmed.ncbi.nlm.nih.gov/25661104/

23. Tinelli R, Malzoni M, Cosentino F, Perone C, Tinelli A, et al. Feasibility, safety, and efficacy of conservative laparoscopic treatment of borderline ovarian tumors. Fertil Steril. 2009; 92: 736-741. PubMed: https://pubmed.ncbi.nlm.nih.gov/18793773/ B

24. Song T, Kimb MK, Jung YW, Yun BS, Seong SJ, et al. Minimally invasive compared with open surgery in patients with borderline ovarian tumors. Gynecol Oncol. 2017; 145: 508-512. PubMed: https://pubmed.ncbi.nlm.nih.gov/28381342/

25. Chen $\mathrm{CH}$, Chiu LH, Chen $\mathrm{HH}$, Chan C, Liu WM. Comparison of robotic approach, laparoscopic approach and laparotomy in treating epithelial ovarian cancer. Int J Med Robot. 2016; 12: 268-275.

PubMed: https://pubmed.ncbi.nlm.nih.gov/25808671/

26. Zhao J, Liu C, Liu J, Qu P. Short-term Outcomes and Pregnancy Rate After Laparoscopic Fertility-Sparing Surgery for Borderline Ovarian Tumors. A Single-Institute Experience. Int J Gynecol Cancer. 2018; 28: 274-278.

PubMed: https://pubmed.ncbi.nlm.nih.gov/29324543/

27. Jiao X, Hu J, Zhu L. Prognostic Factors for Recurrence After FertilityPreserving Surgery in Patients With Borderline Ovarian Tumors. A Systematic Review and Meta-analysis of Observational Studies. Int J Gynecol Cancer. 2017; 27: 1833-1841.

PubMed: https://pubmed.ncbi.nlm.nih.gov/28816706/

28. Lee JH, Kim J. Surgical Techniques of Laparoscopic Omentectomy Springer International Publishing Switzerland 2018737 Alkatout, L. Mettler (eds.), Hysterectomy. 2018.

29. Fauvet R, Boccara J, Dufournet C, Poncelet C, Daraı E. Laparoscopic management of borderline ovarian tumors:results of a French multicenter study. Ann Oncol. 2005; 16: 403-410.

PubMed: https://pubmed.ncbi.nlm.nih.gov/15653700/

30. Shin YJ, Lee HJ, Kim KR, Nam JH, Park JY. Port-site recurrence 6 years after laparoscopic surgery for early stage ovarian borderline malignancy. J Obstet Gynaecol. 2017; 38:2, 291-292. PubMed: https://pubmed.ncbi.nlm.nih.gov/28830247/

31. Radosa JC, Radosa MP, Schweitzer PA, Juhasz-Boess I, Rimbach S, et al. Report of the survey on current opinions and practice of German Society for Gynecologic Endoscopy (AGE) members regarding the laparoscopic treatment of ovarian malignancies. Arch Gynecol Obstet. 2018; 297: 1255-1264.

PubMed: https://pubmed.ncbi.nlm.nih.gov/29520665 